\title{
Super-Arrhenius slowing down in the metastable liquid of hard spheres
}

\author{
M. Dzugutov \\ Department for Numerical Analysis and Computer Science, \\ Royal Institute of Technology, SE-100 44 Stockholm, Sweden
}

(Dated: October 31, 2018)

\begin{abstract}
It is demonstrated that a straightforward extension of the Arrhenius law accurately describes diffusion in the thermodynamically stable liquid of hard spheres. A sharp negative deviation from this behaviour is observed as the liquid is compressed beyond its stability limit, which can be compared with super-Arrhenius slowing down in supercooled liquids. It is suggested that the observed dynamical transition is caused by extra entropy barriers arising in the free-energy landscape. This interpretation is corroborated by the observation of characteristic anomalies in the geometry of a particle trajectory.

PACS numbers: $66.10 . \mathrm{Cb}, 64.70 . \mathrm{Pf}$
\end{abstract}

Computer simulations of liquids using hard spheres (HS) remain a key source of information about the most fundamental aspects of the liquid state. The main intellectual attraction of these models is in their purely geometric nature: all the observable properties of a HS liquid can be reduced to a single thermodynamic quantity - entropy, a measure of finite-range structural correlations [1, 2]. Thus, mapping a phenomenon exhibited by liquids with continuous interactions on a HS model yields its interpretation in terms of the statistical geometry of sphere packing.

An important aspect of liquid behaviour addressed using HS models is supercooled liquid dynamics [3, 4, 5, 6. This notion refers to the complex of dynamical anomalies observed in a liquid which remains in a metastable equilibrium below its melting point. For the one-component HS system, the stable liquid domain is bounded by the critical value of the packing fraction $\eta_{c}=0.493$ [6] ( $\eta=\pi \rho \sigma^{3} / 6$, where $\rho$ is the density and $\sigma$ is the HS diameter). Having been compressed beyond $\eta=0.54$, the HS liquid inevitably crystallizes [6].

The most prominent indicator of the supercooled liquid regime, and, in fact, its defining feature [7, 8], is the characteristic behaviour of the relaxation dynamics which slows down with cooling progressively faster than it can be inferred from the Arrhenius prescription universally describing the temperature variation of the transport coefficients in stable liquids.

In contrast to atomistic models with continuous interaction, the HS systems lack the energy scale. Therefore, in order to compare the non-Arrhenius liquid dynamics as observed in the former with the respective behaviour of the metastable HS liquid, we have to resolve the fundamental problem of finding a description of the stable HS liquid dynamics that would be an adequate analog of the Arrhenius law. In this capacity, Hildebrant-Batchinski relation [9] connecting the diffusion coefficient with the free volume was suggested [10]. The HS liquid diffusion [10] follows this relation in the stable domain and deviates from it for $\eta>\eta_{c}$ [1]. However, this deviation is positive and, therefore, cannot be regarded as an appropriate analog of the super-Arrhenius slowing down in supercooled liquids.

In this Letter, it is demonstrated that an earlier suggested relation between the diffusion coefficient and the thermodynamic entropy [12 can be interpreted as a straightforward extension of the Arrhenius law. Having been tested by the molecular dynamics, it is found to accurately describe the diffusion in the stable onecomponent HS liquid. Moreover, a significant slowing down as compared with this relation is observed in the metastable HS liquid domain, a direct analogue of the super-Arrhenius slowing down in supercooled liquids. This transition in the HS dynamics is discussed in terms of the free energy landscape.

The Arrhenius law asserts that the diffusion coefficient, $D$, scales with the temperature $T$ as

$$
D=D_{0} e^{-A / k_{B} T}
$$

where $A$, the so-called activation energy, is interpreted as the average height of the free-energy barrier, per particle, that has to be crossed in order to perform an elementary step in the process of diffusion. It can be presented as $A=E_{a}-k_{B} T S_{a}$, where $E_{a}$ and $S_{a}$ represent, respectively, the heights of the energy barrier and the entropy barrier involved. For the HS liquid, $A / k_{B} T=-S_{a} ; e^{S_{a}}$ can be understood as the inverse average number of attempts per a successful diffusive transition of a particle. Two possible types of the free-energy landscape can be discussed in the context:

(i) The available configurations are abundantly connected; the connectivity is facilitated by independent motions of individual particles, and each particle has an unrestricted immediate access to the entire space volume assigned to it by the structural constraints. Then the inverse average number of attempts needed for a particle to move from its current position scales as $e^{-A / k_{B} T}=e^{s}$ where $s$ is the excess entropy, per particle, - the difference between the system's entropy and that of the perfect gas at the same thermodynamic conditions. 
(ii) The configuration-space connectivity is restricted by additional constraints, complementary to the ensemble-averaged structural constraints dominating type (i) landscape. In this case, the diffusive motions of an individual particle are coupled, in a presumably hierarchical manner, with the respective motions of other particles within a certain range [13]. These dynamical constraints give rise to extra high entropy barriers which divide the configuration space into a set of components [14]. These barriers dominate the relaxation dynamics and, in this way, control the diffusion rate. In terms of Eq. (1) this means that $e^{-A / k_{B} T}<e^{s}$ It has to be emphasized that this type of free-energy landscape exists on a limited time-scale and, therefore, is quite distinct from the equilibrium distribution. However, there exists a conjecture 15] expressing the cooperativity range in terms of equilibrium entropy; this conjecture is not discussed here.

The described landscape regimes can be understood by keeping in mind an important topological peculiarity of the multidimensional configuration space [16] where each point is adjacent to almost all other points. This can be illustrated by the "small world" topology of abundantly connected multidimensional networks [17]. On the other hand, if an entropy barrier characteristic of type (ii) landscape arises, it will be immediately close to almost all points of the configuration space with a profound effect on the general rate of dynamics.

The central postulate we adopt here is that the freeenergy landscape that controls the relaxation dynamics in the stable HS liquid is of type (i). Having assumed this, Arrhenius law (1) can be transformed into the following simple form relating $s$ and $D$ :

$$
D=D_{0} e^{s}
$$

If the time-scale is measured in terms of the Enskog collision frequency $\Gamma$ [18], this relation becomes equivalent to the earlier suggested scaling law for atomic diffusion [12] which was successfully tested on a number of simple liquids, including the HS liquid using a two-body approximation for $s$ [2]. In the full $s$ version, the scaling law was tested on some metallic liquids [19], but for the HS liquid, such tests have not been done so far. In this context, a conjecture should be mentioned where $D \propto e^{B s}$ with $B \neq 1$ [20].

In this study, relation (2) was tested using a moleculardynamics simulation of a HS system comprising 6912 identical particles. This simulation was necessitated by the fact that, although the HS diffusion has been explored in a number of simulations, a considerable uncertainty remains concerning its size-dependence at different densities [6, 21].

The assumption that the dynamics in a liquid is controlled by the excess entropy, a measure of the structural correlations, implies that the length should be measured in terms of the characteristic length of the structure
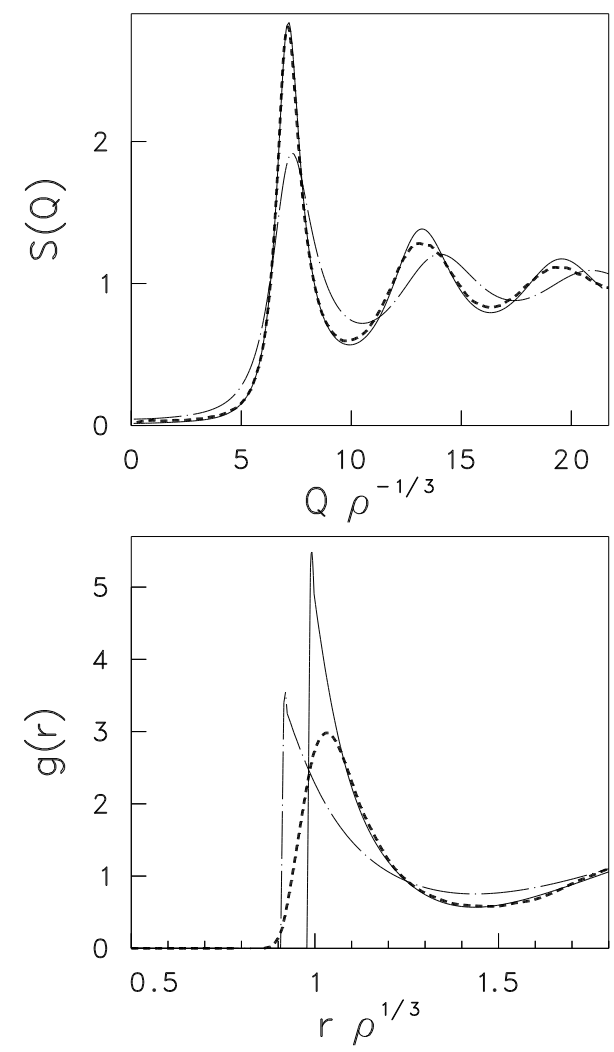

FIG. 1: The structure factor and the radial distribution function of the HS liquid. Solid line, $\eta=0.49$; dash-dotted line, $\eta=0.38$. The dashed line, in both plots, the LennardJones liquid simulated at $\rho=0.85$ and $T=0.7$.

which manifests itself in the position of the main peak of the structure factor. Fig. 1 shows that the latter scales with the density $\rho$ as $\rho^{1 / 3}$; therefore, $\rho^{-1 / 3}$ is a convenient unit of length. Fig. 1 also shows that this unit of length is consistent with the equilibrium separation of the nearest neighbours in the Lennard-Jones liquid of which the structure factor can be reproduced by that of the HS liquid. The use of $\sigma$ as the unit of length that was adopted in the earlier test of the scaling law [12] on the HS liquid is apparently inappropriate at lower densities.

The HS excess entropy was calculated here by the thermodynamic integration using Boublik-Nezbeda approximation 23] for the equation of state. A minor correction was introduced for $\eta>0.47$ [6].

The simulation results are shown in Fig. 2. Each value of $D$ was obtained from the mean-square displacement averaged over $10^{4}$ collisions per particle. These results are compared with another set of the HS diffusion data which represent an extrapolation to the infinite system size 21, 22]. It is clear that with $D_{0}=0.079$, relation (2) accurately describes the diffusion in the one-component HS system within the range $0.36<\eta<0.49$ which covers the entire stable liquid domain.

$D_{0}$ corresponds to the diffusion rate in a hypothetical 


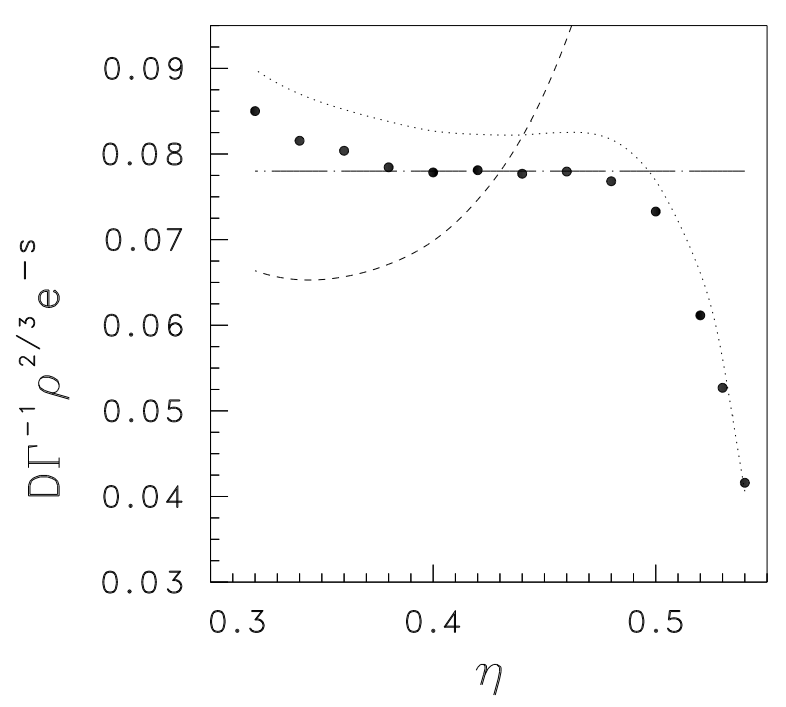

FIG. 2: Diffusion coefficient in the HS liquid as a function of the packing fraction $\eta$. Dots, the present molecular dynamics simulation; dotted line, the extrapolated limit for the infinite system size 21, 22]; chain-dashed line: Eq. (2)XS with $D_{0}=$ 0.078. Dashed line: the Enskog approximation 18].

situation where the discussed liquid diffusion mechanism is still valid but $s=0$. The observed value of $D_{0}$ can be rationalized using the following arguments based on a simple toy model in the spirit of the lattice gas models. Consider a tessellation of the space into equal-size cells. Each cell contains one stationary particle which stays within the cell until its status is changed; besides, there is a number of mobile particles distributed in space. At each time-step, a mobile particle collides with the stationary particle that is in the same cell. As a result, the former becomes stationary, whereas the latter becomes mobile and traverse the cell boundary into a randomly chosen adjacent cell (if several mobile particles simultaneously occur in one cell, an arbitrary collision sequence is assumed). Thus, each particle performs a random walk with the step size $\approx \rho^{-1 / 3}$. Assuming that the number of mobile particles is small, and keeping in mind that there is one particle jump per two collisions, the diffusion coefficient can be estimated as $D \approx \Gamma \rho^{-2 / 3} / 12$, which is in a good agreement with the above value of $D_{0}$.

The results presented in fig. 2 demonstrate that in the metastable liquid domain $\eta>\eta_{c}$, the HS diffusion exhibits a rapidly increasing slowing down as compared with the Arrhenius-like behaviour conjectured by relation (2). Apparently, this behaviour can be regarded as a direct analog of the super-Arrhenius slowing down in the supercooled liquids with continuous interactions. Using the above arguments, the onset of this new dynamical regime can be interpreted as a clear signal that the height of entropy barriers exceeds the ensemble-averaged excess entropy, and the free-energy landscape that controls the

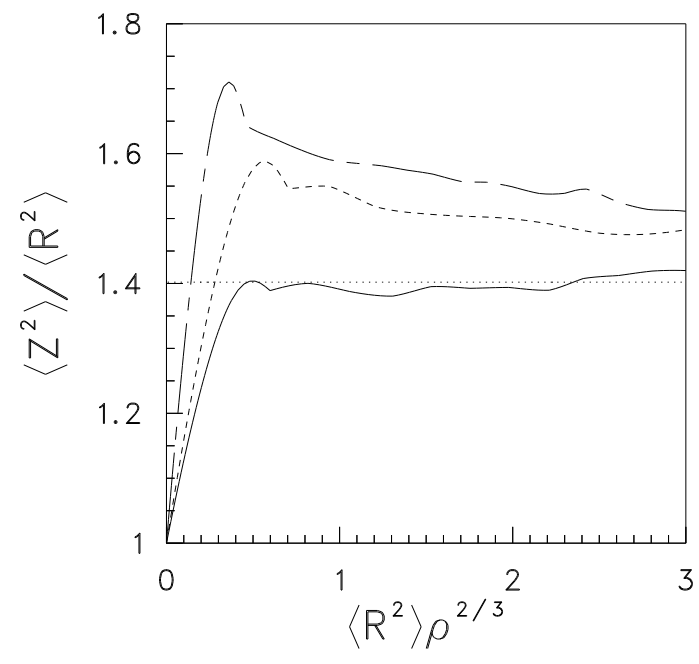

FIG. 3: The ratio of the mean square maximum displacement $\left\langle Z^{2}(t)\right\rangle$ to the mean square displacement $\left\langle R^{2}(t)\right\rangle$. Solid line, $\eta=0.48$; dashed line, $\eta=0.52$; chain-dashed line, $\eta=0.53$. Dotted line indicates the random walk limit $\left\langle Z^{2}(t)\right\rangle /\left\langle R^{2}(t)\right\rangle=$ $1.4024 \ldots 24$.

HS liquid dynamics transforms from type (i) to type (ii).

The long-time configuration-space constraints conjectured to dominate the HS liquid dynamics in the metastable domain can be detected from their impact on a particle trajectory. The latter represents a $3 \mathrm{D}$ real space projection of the multidimensional trajectory of the system in its configurational space. The relaxation dynamics that unfolds in type (i) landscape represents a random walk in the configurational space, which must also be true for a single particle trajectory. The longtime constraints caused by the development of extra barriers characteristic of the type (ii) landscape give rise to distinct anomalies in the geometry of a particle trajectory. A quantity that can be conveniently employed as an indicator of a possible deviation from the random walk geometry in a particle trajectory is the maximum absolute value of the displacement from the original position within the time interval $(0, t)$ :

$$
Z(t) \equiv \max \{R(\tau), \quad 0<\tau<t\}
$$

The ratio of its second moment $\left\langle Z^{2}(t)\right\rangle$, the mean square maximum displacement, to the mean square displacement $\left\langle R^{2}(t)\right\rangle$ for a random walk in $3 \mathrm{D}$ space is equal to $1.4024 \ldots$ 24]. The evolution of this ratio as a function of $\left\langle R^{2}(t)\right\rangle$ for various values of $\eta$ is presented in Fig. 3. For $\eta=0.48$, in the stable liquid domain, the random-walk limit is attained after a short initial period of ballistic behaviour, as soon as a particle leaves the cage of its nearest neighbours. The geometry of a particle trajectory changes significantly as the HS liquid is compressed beyond its stability limit: a new regime of diffusion develops where $\left\langle Z^{2}(t)\right\rangle /\left\langle R^{2}(t)\right\rangle$ exceeds the ran- 
dom walk value. This indicates that a particle trajectory has a higher chance than random to return to an earlier covered region. The extent of this apparent confinement effect in a particle trajectory amounts to the diffusive displacement of several particle diameters, and it increases rapidly, as well as the magnitude of the effect, with the increase of $\eta$.

According to the arguments presented above, the height of an entropy barrier can be regarded as a measure of the real-space extent of the local configurational transformation that the system has to perform to cross the barrier. Extra high barriers in type (ii) landscape correspond to extended correlations, both positional and dynamical. The rapid increase in the (time-limited) correlation length in the metastable HS liquid that has been detected here from the geometry of a particle trajectory represents a generic feature of the supercooled liquid behaviour [25]. Ergodicity restoring relaxation whereby the extra high entropy barriers are created and destroyed is facilitated by highly collective activated hopping. This dynamics was discerned in the metastable HS liquid using dynamical density-functional theory analysis 26].

The profound similarity between the pattern of slowing down in the metastable HS liquid and that in conventional supercooled liquids indicates the entropic nature of this phenomenon 27], and suggests a unifying scenario for its development in terms of the free-energy landscape transformation. The change in the packing geometry under cooling/compression beyond the liquid phase stability limit, presumably caused by reduction of the free volume, leads to formation of long-lived extra high entropy barriers and the loss of the short time-scale configuration-space connectivity. This results in changing the relation between between $s$ and $D$ which can be detected as a deviation from the scaling law (2).

This study was supported by the Swedish Research Council.

[1] J. P. Hansen and I. McDonald, Theory of Simple Liquids, (Academic Press, London, 1976)

[2] R. D. Mountain, and H. Raveche, Journ. Chem. Phys. 35, 2250-2255 (1971)

[3] J. P. Boon and S. Yip, Molecular Hydrodynamics,
McGraw-Hill, New York (1980)

[4] W. Götze, and L. Sjögren, Rep. Progr. Phys., 55, 241 (1992)

[5] E. D. G. Cohen, Physica A, 194 , 229-257 (1993)

[6] R. J. Speedy, Mol. Phys., 95, 168 (1998)

[7] M. D. Ediger, C. A. Angell, and S. R. Nagel, J. Phys. Chem., 100, 13200 (1996)

[8] C. A. Angell, Journ. of Non-Cryst. Solids, 131-133, 13 (1991)

[9] A.J. Batchinski, Z. Phys. Chem., 84, 643 (1913); J.H. Hildebrand, Viscosity and Diffusion, (Wiley, New York, 1977)

[10] L.W. Woodcock, J. Chem. Soc. Faraday Trans. 2, 72, 1667 (1976)

[11] L.W. Woodcock and C.A. Angell, Phys. Rev. Lett., 47, 1129 (1981)

[12] M. Dzugutov, Nature, 381, 137-139 (1996)

[13] R. G. Palmer, D.L. Stein, E. Abrahams, and P.W. Anderson, Phys. Rev. Lett. 53, 958 (1984)

[14] R. G. Palmer, Adv. in Phys. 31, 669 (1982)

[15] G. Adam and J. H. Gibbs, Journ. Chem. Phys. 43, 139 (1965)

[16] S.-K. Ma, Statistical mechanics, World Scientific, Singapore (1985)

[17] D. J. Watts and S. H. Strogatz, Nature, 393, 440 (1998); R.Albert, H. Jeong, and A.-L. Barabasi, Nature, 406, 378 (2000)

[18] S. Chapman and T. G. Cowling, The mathematical theory of non-uniform gases, (University Press, Cambridge, 1939)

[19] J. Hoyt, M. Asta, and B. Sadigh, Phys. Rev. Lett., 85, 594 (2000)

[20] Y. Rosenfeld, Phys. Rev. A 15, 2545 (1977); Y. Rosenfeld, J. Ppys.; Cond. Matt., 11, 5415 (1999)

[21] J. J. Erpenbeck, W. W. Wood, Phys. Rev. A, 43, 4254, (1991)

[22] B.J.Alder, D.M.Gass, and T.E.Wainwright, J. Chem. Phys., 53, 3813, (1991)

[23] T. Boublik and J. Nezbeda, Coll. Czech. Chem. Commun., 51, 2301 (1985)

[24] V. Seshadri and K. Lindenberg, J. Stat. Phys., 22, 69 (1980)

[25] E. W. Fischer, E. Donth, and W. Steffen, Phys. Rev. Lett. 68, 2344 (1992); W. Kob, C. Donati, S. J. Plimpton, P. H. Pool, and S. C. Glotzer, Phys. Rev. Lett. 79, 2827 (1997); E. R. Weeks, J. C. Crocker, A. C. Levitt, A. Scofield, and D. A. Weitz, Science, 287, 627 (2000)

[26] K. Fuchizaki, K. Kawasaki, J. Phys. Soc. Japan, 67, 2158, (1998)

[27] A. Crisanti and F. Ritort, cond-mat/0102104 (2001) 